\title{
The expanding role of incretin-based therapies: how much should we expect?
}

\section{Martin Haluzik}

Third Department of Medicine, First Faculty of Medicine, General University Hospital, Charles University, Prague, Czech Republic
Correspondence should be addressed to $\mathrm{M}$ Haluzik Email mhalu@If1.cuni.cz
The increasing prevalence of type 2 diabetes mellitus (T2DM) worldwide and its combination with other pathologies including obesity, arterial hypertension, and dyslipidemia make this disorder one of the most significant challenges of the 21st century. Although multiple classes of antidiabetic drugs are available, more than half of the patients with T2DM still do not achieve optimal glucose control owing to the progressive nature of T2DM requiring stepwise therapy intensification and suboptimal compliance to diet and lifestyle measures as well as side effects of most antidiabetic drugs, in particular, hypoglycemia and weight gain. An urgent need for safer and more effective treatments has stimulated the development of several novel classes of antidiabetic drugs in the last decade with promising new molecules still emerging (Mraz et al. 2009). In contrast to the traditional glucocentric view, the focus has shifted toward a more complex approach emphasizing the importance of the beneficial effects of novel antidiabetics on multiple cardiovascular risk factors (Skyler et al. 2009). The discovery of incretin-based therapies has markedly improved our possibilities of T2DM treatment, owing not only to their good antidiabetic efficacy but also to other positive effects such as reduction of body weight and blood pressure and improvement of dyslipidemia as well as their potential neuroprotectivity and cardioprotectivity. In this miniseries, we have put together four reviews from leading research groups in the field focusing on some of the most intensively discussed effects of incretin-based therapies beyond the improvement of glucose control.

The incretins are peptide hormones released from the small intestine during meal absorption that stimulate insulin secretion from pancreatic $\beta$-cells and reduce excessive secretion of glucagon by pancreatic $\alpha$-cells, thus improving two important defects of T2DM (Holst et al. 2008). Of the two currently known incretins, glucagon-like peptide 1 (GLP1) and glucose-dependent insulinotropic polypeptide, only therapies based on the effects of the former are used in clinical practice. Two approaches have been adopted based either on the prolongation of the halflife of endogenous GLP1 by blocking its degradation by dipeptidyl peptidase 4 (DPP4) (DPP4 inhibitors) or on the stimulation of GLP1 receptor (GLP1R) with compounds resistant to DPP4 degradation (GLP1R agonists).

One important effect of GLP1 and GLP1R agonists observed in both preclinical and clinical studies was the reduction of food intake and body weight, which makes these medications rather unique within the antidiabetic armamentarium. In the first review of this miniseries, van Bloemendaal et al. (2014) discuss the effects of GLP1 and GLP1R agonists on appetite and body weight, focusing on their potential energy balance-regulating effects in the CNS. Although the fact that GLP1 and GLP1R mimetics have consistent acute effects on hunger and satiety has been known for some time, the underlying mechanisms are still only partially understood. They may include the well-documented delayed gastric emptying as well as potential direct effects in the CNS. The mechanism behind the effects is complex and clearly differs among the short-acting and the long-acting GLP1R agonists. These findings suggest that other components such as the direct action of long-acting GLP1R agonists might be in place.

Cardiovascular side effects have been a major concern of several antidiabetic and antiobesitic medications, eventually leading to the withdrawal of a number of drugs from the market. Therefore, the antidiabetic medications are currently being intensively scrutinized for cardiovascular safety. Preclinical and small clinical studies have shown that GLP1 has numerous beneficial effects on the cardiovascular system, including the reduction of blood pressure, the improvement of lipid profile, the amelioration of endothelial dysfunction, and

Published by Bioscientifica Ltd.

This editorial accompanies the thematic review special issue on Incretins. The Guest

Editor for this section was Martin Haluzik, Charles University Braque, Gzech Republic. $_{1}: 39: 16 \mathrm{AM}$ 
even direct cardioprotection in the experimental models of ischemia/reperfusion. The first clinical studies suggesting the effects of GLP1R agonists on ischemic preconditioning have recently been published. The important topic of cardiovascular effects of incretin-based therapies and their potential underlying mechanisms is covered in the review by Angeli \& Shannon (2014).

Although T2DM certainly presents a huge global threat to mankind, neurodegenerative diseases are beyond all doubt a global peril to the aging population. It has been demonstrated more than a decade ago that insulin resistance is by far not limited to peripheral tissues such as the liver, the muscle, and the fat, but that it is also occurs in the brain (Bruning et al. 2000). Along this line, T2DM increases the risk of neurodegenerative diseases such as Parkinson's disease and Alzheimer's disease. GLP1Rs are widely distributed within the brain, and experimental studies have suggested that they may also protect against neurodegeneration by multiple mechanisms. This important topic is the focus of the review by Dr Holscher (2014), one of the pioneers in this field.

The topic of the final review of the miniseries is the pancreatic safety of incretin-based therapies. Some preclinical studies have indicated that chronic GLP1R activation may stimulate the proliferation of pancreatic $\beta$-cells and thus increase $\beta$-cell mass, providing hope that this approach may potentially alter the natural progression of T2DM (Sturis et al. 2003). On the contrary, other studies have raised concerns that chronic use of incretin-based therapies may increase the risk of pancreatitis and pancreatic cancer (Matveyenko et al. 2009). The discussion has been further stimulated by the publication of human morphological data pointing to increased pancreatic ductal proliferation in patients treated with incretin-based therapies (Butler et al. 2013). Although the methodology described in the latter paper has been widely criticized, the potential pancreatic side effects of incretinbased therapies remain certainly an open and widely discussed topic. The difficult task of reviewing this subject has been undertaken by Lamont \& Andrikopoulos (2014).

In summary, it is certainly safe to say that incretinbased therapies have markedly broadened our armamentarium of antidiabetic therapies. Nonetheless, as with all novel therapies, the potential hopes as well as fears can only be clarified by carefully performed clinical trials. In the meanwhile, we sincerely hope that our miniseries on incretin-based therapies can help both the researchers and the clinicians to better understand this rapidly evolving field.

\section{Declaration of interest}

The author declares that there is no conflict of interest that could be perceived as prejudicing the impartiality of this editorial.

\section{Funding}

The authors' research was supported by Research Project RVO-VFN-64165 of the Ministry of Health of Czech Republic.

\section{References}

Angeli FS \& Shannon RP 2014 Incretin based therapies: can we achieve glycemic control and cardioprotection? Journal of Endocrinology 221 T17-T30. (doi:10.1530/JOE-13-0195)

van Bloemendaal L, Ten Kulve JS, la Fleur SE, Ijzerman RG \& Diamant M 2014 Effects of GLP-1 on appetite and body weight: focus on the central nervous system. Journal of Endocrinology 221 T1-T16. (doi:10.1530/ JOE-13-0414)

Bruning JC, Gautam D, Burks DJ, Gillette J, Schubert M, Orban PC, Klein R, Krone W, Muller-Wieland D \& Kahn CR 2000 Role of brain insulin receptor in control of body weight and reproduction. Science $\mathbf{2 8 9}$ 2122-2125. (doi:10.1126/science.289.5487.2122)

Butler AE, Campbell-Thompson M, Gurlo T, Dawson DW, Atkinson M \& Butler PC 2013 Marked expansion of exocrine and endocrine pancreas with incretin therapy in humans with increased exocrine pancreas dysplasia and the potential for glucagon-producing neuroendocrine tumors. Diabetes 62 2595-2604. (doi:10.2337/ db12-1686)

Holscher C 2014 Central effects of GLP-1: new opportunities for treatments of neurodegenerative diseases. Journal of Endocrinology 221 T31-T41. (doi:10.1530/JOE-13-0221)

Holst JJ, Deacon CF, Vilsboll T, Krarup T \& Madsbad S 2008 Glucagon-like peptide-1, glucose homeostasis and diabetes. Trends in Molecular Medicine 14 161-168. (doi:10.1016/j.molmed.2008. 01.003)

Lamont B \& Andrikopoulos S 2014 Is there preclinical evidence that incretin-based therapies alter pancreatic morphology? Journal of Endocrinology 221 T43-T61. (doi:10.1530/JOE-13-0557)

Matveyenko AV, Dry S, Cox HI, Moshtaghian A, Gurlo T, Galasso R, Butler AE \& Butler PC 2009 Beneficial endocrine but adverse exocrine effects of sitagliptin in the human islet amyloid polypeptide transgenic rat model of type 2 diabetes: interactions with metformin. Diabetes 58 1604-1615. (doi:10.2337/db09-0058)

Mraz M, Bartlova M, Lacinova Z, Michalsky D, Kasalicky M, Haluzikova D, Matoulek M, Dostalova I, Humenanska V \& Haluzik M 2009 Serum concentrations and tissue expression of a novel endocrine regulator fibroblast growth factor-21 in patients with type 2 diabetes and obesity. Clinical Endocrinology 71 369-375. (doi:10.1111/j.1365-2265.2008. 03502.x)

Skyler JS, Bergenstal R, Bonow RO, Buse J, Deedwania P, Gale EA, Howard BV, Kirkman MS, Kosiborod M, Reaven P et al. 2009 Intensive glycemic control and the prevention of cardiovascular events: implications of the ACCORD, ADVANCE, and VA Diabetes Trials: a position statement of the American Diabetes Association and a Scientific Statement of the American College of Cardiology Foundation and the American Heart Association. Journal of the American College of Cardiology 53 298-304. (doi:10.1016/ j.jacc.2008.10.008)

Sturis J, Gotfredsen CF, Romer J, Rolin B, Ribel U, Brand CL, Wilken M, Wassermann K, Deacon CF, Carr RD et al. 2003 GLP-1 derivative liraglutide in rats with $\beta$-cell deficiencies: influence of metabolic state on $\beta$-cell mass dynamics. British Journal of Pharmacology 140 123-132. (doi:10.1038/sj.bjp.0705397)

Published by Bioscientifica Ltd 